\title{
Physics-based simulation models for digital twin development in laser powder bed fusion
}

\section{Lihang Yang and Tuğrul Özel*}

\author{
Department of Industrial and Systems Engineering, \\ Manufacturing and Automation Research Laboratory, \\ Rutgers University, \\ Piscataway, New Jersey, 08854, USA \\ Email: 1y189@scarletmail.rutgers.edu \\ Email:ozel@rutgers.edu \\ ${ }^{*}$ Corresponding author
}

\begin{abstract}
The digital twin for metal additive manufacturing is under development by unifying all simulation models integrated with the sensory observations for bi-directional data flow powered by machine learning. This paper presents physics-based simulation modelling for predicting the thermal field solution and molten pool geometry in laser powder bed fusion (L-PBF) process for Ti6A14V, SS316L, and IN625 metal powders. Two-dimensional (2D) thermal field on the powder bed surface along the laser path and the hatch direction are computed for a moving laser heat source using implicit numerical formulation to understand the temperature rise during L-PBF process. The temperature field into the powder is computed using the temperature solution obtained along the laser path. The results are compared against the literature to corroborate the computed molten pool geometry. This numerical solution approach is found to be highly practical for computing thermal fields and suitable for digital twin development in metal additive manufacturing.
\end{abstract}

Keywords: additive manufacturing; physics-based simulation; digital twin; powder bed fusion; metal; finite element method; titanium; steel; nickel.

Reference to this paper should be made as follows: Yang, L. and Özel, T. (2021) 'Physics-based simulation models for digital twin development in laser powder bed fusion', Int. J. Mechatronics and Manufacturing Systems, Vol. 14, No. 2, pp.143-163.

Biographical notes: Lihang Yang is a graduate student and received BS in Physics and MS in Industrial and Systems Engineering degrees from Rutgers University in 2017 and 2020 respectively. In 2018, he joined the Manufacturing and Automation Research Laboratory at Rutgers University for his graduate studies. His research interests include additive manufacturing, precision machining, and surface integrity.

Tuğrul Özel received his $\mathrm{PhD}$ in Mechanical Engineering from Ohio State University in 1998, a BS from Aeronautical Engineering from Istanbul Technical University, Turkey in 1987. He is a Full Professor with the Industrial and Systems Engineering Department of Rutgers University and the Director of Manufacturing and Automation Research Laboratory (MARL). He has over 150 peer-reviewed journal and conference papers. He serves as an Editor-inChief for International Journal of Mechatronics and Manufacturing Systems, 
an Associate Editor for the ASME Journal of Manufacturing Science and Engineering Control and a member of several editorial boards of international journals. He is a Senior Member of ASME, ASPE, and SME.

\section{Introduction}

Laser fusion-based metal additive manufacturing requires complete process understanding to achieve production of high-quality parts with consistency. In order to optimise a particular L-PBF process for a given part design, its progression from microscale to macro-scale through the full processing steps must be considered and understood, which can be achieved through the use of validated physics-based simulation models. By linking these models together and providing them with sensory measurement data from physical part manufacturing, it is possible to create a 'digital twin' of the entire L-PBF process towards a digital twin for the part production scale. Notwithstanding, creation of such a comprehensive multi-scale digital twin would be a costly and time-consuming effort, it would support in achieving the full advantages of fusion-based AM in production environments while increasing productivity and cost-effectiveness.

Physics-based process simulations and simulation-based engineering has the potential to speed up the development of complex additive manufacturing systems such as modern L-PBF machines in the industry. Machine learning as an essential tool for analysing datasets offers potentials to identify meaningful patterns in the large amounts of data being collected from complex AM systems and develop digital twins for L-PBF processes. However, simplified physics-based models are urgently needed to make the digital twin predictive and useful. Another innovation is new hardware architectures that allow users to collect and analyse data efficiently to then incorporate the data into digital twins. As computer modelling and digital twins are becoming increasingly important, simple physics-based simulation models for the L-PBF process provide insight on the physics of the manufacturing process. Gaikwad et al. (2020) provided a comprehensive review of research work toward obtaining the digital twin for additive manufacturing processes including L-PBF. Their work identifies four fields important for the digital twin paradigm including real-time process monitoring, physics-based simulation modelling, in-situ sensing, and data analytics (machine learning). Mukherjee and DebRoy (2019) identified the need to develop various simulation models covering physical phenomena involve with metallic 3D printing processes that are validated with in-situ process data for dissimilar alloys at different processing settings.

Simulation models at multiple-length scales are required as illustrated in Figure 1. At micro-scale the laser-powder material interaction results in a melt pool formation, spatter ejection, keyhole and other liquid melt pool flow effects which is the most fundamental physics-based simulation model. At macro-scale layerwise construct should be modelled and simulated by linking the melt pool scale models into full layer processing. This should be linked to layer-by-layer development of 3D part construct and the complete signature of the process on the final part built should be determined. The cross-validation of simulation models with the sensory measurements and data must be incorporated by using machine learning methods for calibrating and optimising process simulation parameters. This approach should be tested and certified for powder materials 
used in metal AM processes such as steels, titanium and nickel-based alloys and other materials for creating a material database for processing-property-structure relationship knowledge collection.

Figure 1 The concept of physics-based simulations and digital twin for L-PBF AM processes (see online version for colours)

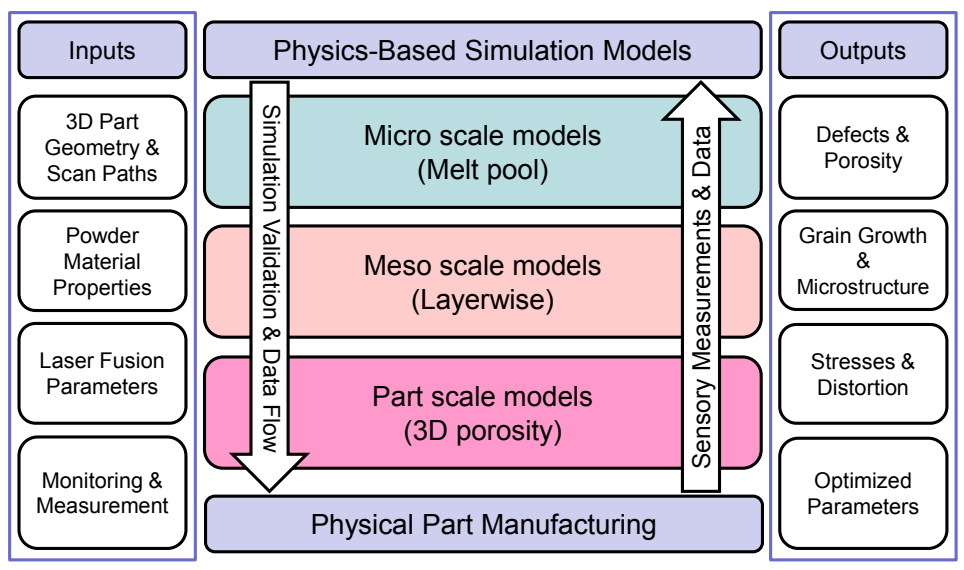

Metal parts fabricated using powder materials in stainless steels, titanium, and nickel alloys using L-PBF based methods have been widely investigated. A high-power laser beam processes the surface of the powder metal material one layer at a time in an inert gas filled chamber to build 3D fused metal parts. Such high-power laser processes are complicated to control for the reason that rapid heating and cooling rates involved with problems including incomplete fusion, keyholing, and formation of residual stress and warpage. Therefore, the calculation of temperature profile and molten pool geometry in L-PBF process has been a research interest to the user of metal additive manufacturing due to the importance in fabrication of metal parts with consistent quality.

Accurate powder material properties and input parameters for the L-PBF process are need alongside with a good understanding of the working principles of the laser-based powder fusion for complete physics-based simulation models. The working principles of the L-PBF and input parameters are illustrated in Figure 2.

Most basic parameter to consider in L-PBF is the energy density induced by laser processing of the powder material. The surface energy density consists of laser power per processed area under the moving laser beam can be given as in equation (1).

$$
E=\frac{P}{v_{s} h}
$$

where $P$ is the laser power, vs is scan velocity, and $h$ is hatch distance. This energy density on powder surface directly relates to the area of heat affected zone, the geometry and the size of the melt pool among other things including depth of fusion and re-melting zone during L-PBF process.

The metal powder used in L-PBF machines has also certain limitations and effects on the process outcomes. Typically, L-PBF machines are equipped with certain ambient inert gas types (e.g., argon, nitrogen, or argon-helium mixtures) available in their configuration for minimising the effects of metal oxidation. For instance, nitrogen is 
utilised for processing stainless steel and nickel alloyed powder material where Argon is utilised for processing titanium alloys due their high chemical affinity with oxygen and nitrogen (Pauzon et al., 2020).

Figure 2 The illustration of the basic set of L-PBF process parameters and variables (see online version for colours)

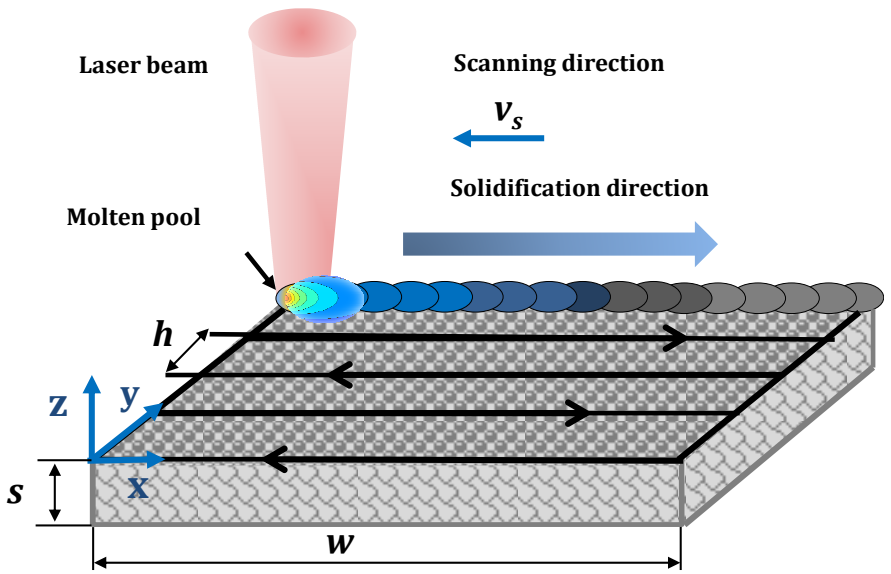

Source: Özel et al. (2018)

In addition, unique powder material properties including average size of the powder grains and varying spreading and packing density to begin with the L-PBF process. Process parameters also varies in a range depending on the L-PBF equipment specs. As an example, Table 1 summarises properties of the powder metallic materials used in L-PBF processes as adopted by other research work appeared in literature where dpowder is average powder particle diameter, $P$ is laser power, $\lambda$ is wavelength, $d$ is spot diameter, vs is scan velocity, $h$ is hatch distance and $\mathrm{s}$ is layer thickness. The alloy materials are stainless steel 316L, nickel-based alloy Inconel 625 (IN625), and titanium alloy (Ti6Al4V).

Table 1 Some L-PBF parameters used for a few metal powder materials

\begin{tabular}{|c|c|c|c|c|c|c|c|c|}
\hline References & Alloy & $\begin{array}{c}\text { dpowder } \\
{[\mu \mathrm{m}]}\end{array}$ & $P[\mathrm{~W}]$ & $\lambda[\mathrm{nm}]$ & $d[\mu \mathrm{m}]$ & $v s[\mathrm{~m} / \mathrm{s}]$ & $h[\mu \mathrm{m}]$ & $s[\mu \mathrm{m}]$ \\
\hline $\begin{array}{l}\text { Anam et al. } \\
(2013)\end{array}$ & IN625 & 37 & $200-120$ & 1060 & 100 & $0.7-1.1$ & 100 & $20-40$ \\
\hline $\begin{array}{l}\text { Yadroitsev } \\
\text { et al. (2007) }\end{array}$ & IN625 & 20 & 50 & 1075 & 70 & 0.13 & $60-140$ & $20-60$ \\
\hline $\begin{array}{l}\text { Criales and } \\
\text { Özel (2017) }\end{array}$ & IN625 & 35 & 169-195 & $1060-1080$ & 90 & $0.725-0.875$ & 100 & 20 \\
\hline $\begin{array}{l}\text { Gusarov } \\
\text { et al. (2007) }\end{array}$ & SS316L & 20 & 30 & 1060 & 60 & $\begin{array}{c}0.12,0.16 \\
0.24\end{array}$ & - & 50 \\
\hline $\begin{array}{l}\text { Hussein } \\
\text { et al. (2013) }\end{array}$ & SS316L & - & 100 & - & 150 & $\begin{array}{c}0.10,0.20 \\
0.30\end{array}$ & - & 1000 \\
\hline $\begin{array}{l}\text { Röttger } \\
\text { et al. (2020) }\end{array}$ & SS316L & 38 & 136.1 & $1070 \mathrm{~nm}$ & 100 & 0.928 & & 40 \\
\hline
\end{tabular}


Table 1 Some L-PBF parameters used for a few metal powder materials (continued)

\begin{tabular}{|c|c|c|c|c|c|c|c|c|}
\hline References & Alloy & $\begin{array}{c}\text { dpowder } \\
{[\mu \mathrm{m}]}\end{array}$ & $P[\mathrm{~W}]$ & $\lambda[\mathrm{nm}]$ & $d[\mu \mathrm{m}]$ & $v s[\mathrm{~m} / \mathrm{s}]$ & $h[\mu \mathrm{m}]$ & $s[\mu \mathrm{m}]$ \\
\hline $\begin{array}{l}\text { Abolhasani } \\
\text { et al. (2019) }\end{array}$ & SS304 & - & 200 & 1064 & 80 & 0.732 & & 30 \\
\hline $\begin{array}{l}\text { Roberts } \\
\text { et al. (2009) }\end{array}$ & Ti6Al4V & 30 & 120 & 1060 & 100 & 0.22 & - & 30 \\
\hline $\begin{array}{l}\text { Fischer } \\
\text { et al. (2002) }\end{array}$ & $\mathrm{Ti}$ & 30 & 3 & 1060 & 100 & 0.001 & - & - \\
\hline $\begin{array}{l}\text { Song et al. } \\
\text { (2012) }\end{array}$ & Ti6Al4V & 30 & 110 & $1064-1100$ & 34 & $0.2-0.6$ & - & 50 \\
\hline $\begin{array}{l}\text { Liu et al. } \\
\text { (2021) }\end{array}$ & Ti6Al4V & 38 & $50-250$ & $1030-1080$ & 100 & $0.2-2$ & 100 & $30-60$ \\
\hline
\end{tabular}

\section{Physics-based modelling of L-PBF processes}

Physics-based modelling effort in L-PBF processing of metallic powder materials have been focused on the process- properties relationship. Particular interests included understanding the influence of heating, melting, and cooling rates on the resulting melt pool geometry, the degree of fusion and mechanical properties (e.g., tensile strength) of the build structures. Specifically, the time exposure of the molten metal pool above the liquidus temperatures (i.e., heated spot) during L-PBF process can be correlated to defect, anomaly, vapour and pore formation which subsequently take the lead into sacrificed material properties of the $3 \mathrm{D}$ build. For this reason, transient thermal field and distribution of temperature distribution throughout the L-PBF processing of the metallic powder surface gain significant importance. A modelling framework is presented in Figure 3 that shows the necessary input parameters about the fusion process variables, laser parameters, powder material and thermal properties for thermal melting model, liquid melt pool model, as well as solidification microstructure models. Input laser parameters include laser power $(P)$, wavelength $(\lambda)$, spot diameter $(d)$, and pulse repetition rate (PRR). Fusion process parameters include scan velocity (vs), hatch distance $(h)$, and scan strategy rotation (SRR). Powder material properties include average powder particle diameter (dpowder), bulk density $(\rho)$, powder density model parameters $(\tau, \gamma, \beta)$, and reflectivity $(R)$. Thermal melting model parameters include thermal properties specific heat of powder and liquid forms $(\mathrm{Cp}, \mathrm{Cl})$, enthalpy $(H)$, conductivity $(k)$, latent heat of fusion (Lf). The outcome of the framework is to obtain a thermal field solution with time-dependent history, thermal gradients, liquid melt pool related solidification and microstructure (grain size and fractions) along with part density, porosity, fusion defects, residual stresses and distortions.

Physics-based thermal simulation models and accurately calculating the temperature distribution during the process are essential to obtaining digital twin for the physical $3 \mathrm{D}$ build counterparts. Most preferred approach for defining and obtaining transient temperature fields for L-PBF is based on numerical solution techniques using finite element method (FEM) for solving heat-conduction-convection equations by considering moving heat source during L-PBF processes as widely reported in the literature. 
Figure 3 The framework for physics-based simulation modelling for L-PBF processes (see online version for colours)

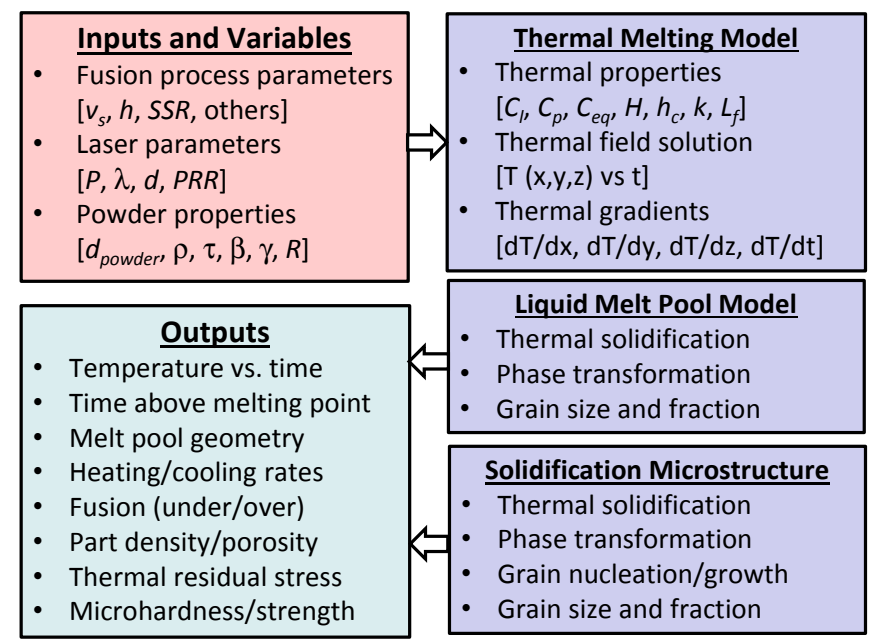

Kolossov et al. (2004) proposed a thermal simulation model using FEM-method with temperature-dependent conductivity and specific heat. Patil and Yadava (2007) used Galerkin-based FEM to analyse the laser induced thermal field and 2D temperature distribution on the single layer titanium powder bed and extended their work to processing single layer stainless steel powder (316L). Gusarov and Smurov (2009) proposed a thermal diffusion coupled kinetics-based simulation model that includes combined considering of radiation, heat diffusion, and powder consolidation for L-PBF. A 3D FEM model for thermal field was first proposed by Roberts et al. (2009) in multilayer laser fusion of titanium alloy Ti-6Al4-V powder material. Yin et al. (2012) proposed thermal FEM-based simulations for the L-PBF process where a Gaussian laser beam, latent heat for fusion, and material enthalpy was used implemented. They used their simulation models to analyse the effect of laser processing on the thermal field and melt pool geometry when beam diameter, power, and scan velocity are varied. Jahn et al. (2012) formed an FEM-based thermal model by implementing Navier-Stokes equations for solid-to-liquid and liquid-to-solid phase transitions to obtain a recurrent melt flow and melt pool boundaries. Vasquez et al. (2012) utilised a 3D FEM model to evaluate laser beam and powder material interaction. Their model produced results helpful for understanding the multi-physics and multi-scale effects. Song et al. (2012) presented FEM-based thermal simulations and conducted process parameter investigations for L-PBF of Ti6Al4V material. Yin et al. (2012) utilised FEM-based simulation models to determine temperature on a single layer powder material. Anam et al. (2013) recommended constructive steps for FEM-based simulation modelling for nickel-based alloy IN625. Yadroitsev et al. (2014) measured thermal field and melt pool size on the Ti6Al4V powder surface and investigated the effect of heat intensity on the resultant microstructure. Denlinger et al. (2016) used 3D FEM-based thermal simulations for calculating residual stress and distortions. Criales et al. (2016) developed successful simulation models using a 2D FEM approach for predicting thermal field during L-PBF 
of IN625 material. Khairallah et al. (2016) offered a 3D physics-based simulation model through computational fluid dynamics combined with heat transfer for molten material flow and particle spatter in the L-PBF of Ti6Al4V material. Criales and Özel (2017) utilised a numerical solution method for the $1 \mathrm{D}$ thermal equation to determine temperature profiles in the L-PBF processing of Ti6Al4V powder. Later, Criales et al. (2017a, 2017b) proposed 2D FEM simulation models to compute thermal field, melt pool size and shape as well as heating, melting, and cooling rates during the L-PBF process on the IN625 powder material layers. Arisoy et al. (2019) employed physics-based 3D FEM models for simulating melt pool and temperature vectors as well as nucleation and solidification during the L-PBF of IN625 powder material in a multi-track processing solution. Schoinochoritis et al. (2017) provided a critical review of FEM models used for metal powder additive manufacturing processes. None of these works present a simulation system with a large material database that is suitable for a digital twin of thermal-physical behaviour of L-PBF. This knowledge gap remains to be filled by offerings of simulation tools with a goal of bringing them together into a digital twin system for the L-PBF.

This study sets the framework of a simulation systems by simply considering the $2 \mathrm{D}$ heating and fusion of powder metal with a laser beam during single-track processing that is formulated by a numerical program developed in MATLAB. At first the 2D thermal simulation is solved and moderately prolonged to $3 \mathrm{D}$ thermal simulation through two $2 \mathrm{D}$ projection thermal fields of a $3 \mathrm{D}$ thermal field. The result is a fuller investigation of the temperature field on the powder bed surface horizontally and beneath the powder bed vertically. This physics-based simulation model provides two distinct outcomes as part of the modelling framework discussed in Figure 3. The first outcome is the time history of the melt pool region and its vicinity, and the second outcome is the overmelting time on the powder bed surface beneath the laser beam.

Another benefit of the proposed model is related to being able to adjust the model parameters and solutions in an open-code platform in Matlab rather than following strict solution procedures available in FEM analysis software packages. Current capabilities of commercially available software packages are indeed limited in development and deployment for predicting thermal/liquid melt pool fields as well as simulating the solidification microstructure. Often unconnected/unlinked simulation models are developed using separate software thermal, fluid dynamics, and microstructure software packages. For this reason, this presented work paves the foundation for open-architecture software tools for physics-based simulation models towards digital twin in metal AM processes.

The contribution of this work is not about comparing the proposed model to the ones existing in the literature. There is a plethora of 2D and 3D thermal models developed in Matlab applied to L-PBF processes available in literature most recently analytical models offered for Ti6Al4V by Liu et al. (2021) and Ökten and Biyikoglu (2021) and for IN625 by Ning et al. (2019) for example. However, these models are not proposed for development of digital twin for the L-PBF thermal processes in mind. They are usually valid for a single powder work material (i.e., Ti6Al4V), although they can be extended. The contribution of this work rather is that the proposed physics-based model validated for Ti-6Al-4V, SS316L, and IN625 will pave a foundation for digital twin development for the physical L-PBF processes. 


\subsection{Physics-based thermal simulation model}

The preliminary mission is to determine physics-based thermal models that can be applied to compute the thermal field during laser-powder material interaction in L-PBF processes. Finding thermal fields is important to properly explain the impacts of rapid heating and cooling intervals on the surface and beneath the powder layer. The thermal diffusion for laser processing can be analysed using a mathematical method, by solving the differential heat balance. Previous research explored a basic 1D heat conduction equation model for computing thermal field into the bed direction of the powder by employing an FDM-based Crank-Nicolson scheme as presented in Criales and Özel (2017).

Similarly, computational problem can be formulated by beginning with solved following heat convection-diffusion equation when heat convection arising from the laser-material interaction to the atmosphere and the radiation emitted by the ambient are considered negligible heat losses during intense heating provided by the laser beam energy density.

$$
\rho C_{p} \frac{\partial T}{\partial t}=\frac{\partial}{\partial x}\left(k \frac{\partial T}{\partial x}\right)+\frac{\partial}{\partial y}\left(k \frac{\partial T}{\partial y}\right)+\frac{\partial}{\partial z}\left(k \frac{\partial T}{\partial z}\right)+q
$$

This the definition for a 3D heat transfer situation where the numerical solution is not only computationally difficult but also time consuming. However, using sufficient assumptions, this can be reduced to a $2 \mathrm{D}$ heat transfer model where the temperature $T$ is considered as function of time $t$ and spatial variables of $x$ and $y$ are representing a 2D field where $x$ is the spatial variable along longitudinal laser scanning direction and $y$ is the spatial variable along the lateral hatching direction (see Figure 2).

$$
\rho C_{p} \frac{\partial T}{\partial t}=\frac{\partial}{\partial x}\left(k \frac{\partial T}{\partial x}\right)+\frac{\partial}{\partial y}\left(k \frac{\partial T}{\partial y}\right)+q
$$

Finally, the laser heat source represented with $q$ is considered as independent of the spatial variable $z$ into powder bed direction. For instance, at a location of $z=0$, the thermal field can be considered at the powder layer surface where transferring this heat concentration into its vicinity and the powder bed direction can only occur through heat conduction.

\subsection{Powder properties}

The properties of powder material with certain packing density such conductivity, specific heat, emittivity, and reflectivity etc. are usually not known where physical and thermal properties of the bulk material are known and oftentimes as function of temperature. It is a reasonable assumption to make that powder metals can follow a linearly varying thermal properties and after reaching to their melting point a phase change occurs for liquid transition.

The effect of the packing density on the material properties can be captured using the following model considering the fraction of the density or porosity $(\tau)$ as trapped air or gas in the powder material density:

$$
\rho_{\text {powder }}=(1-\tau) \rho_{\text {bulk }}
$$


Similarly, conductivity of the powder material can be represented as a fraction of bulk material thermal conductivity:

$$
k_{\text {powder }}=(1-\tau) k_{\text {bulk }}
$$

The specific heat is modelled in a way to capture this thermal property within the molten material region as well as the phase transition from solid to liquid in the simulation model. Since powder metal alloys studied in this research do follow isothermal phase change at a specific temperature but rather follow a net change in enthalpy in between the solidus and liquidus ( $\mathrm{Ts}$ and $\mathrm{Tl}$ ) temperature ranges, an equivalent specific heat can be formulated as given in equation (6).

$$
C_{e q}(T)=\left\{\begin{array}{c}
C_{p}(T), T \leq T_{s} \\
C_{p}(T)+\frac{L_{f}}{T_{l}-T_{s}}, T_{s}<T<T_{l} \\
C_{l}, T \geq T_{l}
\end{array}\right.
$$

where the specific heat of the powder material in solid and in liquid phases are $\mathrm{Cp}(\mathrm{T})$ and $\mathrm{Cl}$ respectively, the solidus and liquidus temperatures are Ts and $\mathrm{Tl}$ respectively, and the latent fusion heat is Lf. This specific heat representation provides continuity between solidus and liquidus regions (Van Elsen et al., 2007; Song et al., 2012).

\subsection{Laser beam as heat source}

The laser beam provides a surface heat source to the powder material that can be modelled using a focused Gaussian beam intensity distribution on the XY surface. The moving heat source by following Gaussian beam intensity equation can be written as in equation (7).

$$
q=(1-R) \frac{2 P}{\pi w_{o}^{2}} e^{-2\left(r^{2} / w_{o}^{2}\right)}
$$

where the laser power, $P$, the reflectivity index, $R$, Gaussian beam waist size, wo, and the beam distance from the centre, $r$ are used. The waist size is the distance at which the laser reaches $1 / e$ of its peak power and varies based on the experimental setup. The laser beam intensity is absorbed merely on the powder bed surface in XY simulation model.

The heat source for the $\mathrm{XZ}$ simulation model considers fixed spatial variable, $y$, and is purely distributed along the spatial variable, $x$. The XY simulation model is concerned with the powder layer surface ( $z=0$ location). Whereas the $\mathrm{XZ}$ simulation is concerned with the centreline of the laser path ( $y=0$ location). A specific condition is set that at the intersection of $\mathrm{XY}$ and $\mathrm{XZ}$ fields the temperature has to have the same value when solving the numerical formulation for the XY model first and then using the resultant temperature profile as the input field to obtain the solution for the $\mathrm{XZ}$ simulation model.

\subsection{Finite element formulation}

A numerical finite element formulation scheme is utilised to represent a layer of powder material with a mesh and compute the thermal field by using the heat conduction solution. A finite element technique proposed by Bergheau and Fortunier (2008) is 
adopted to find an approximate solution to a boundary value problem for a partial differential equation by portioning the solution space into sub elements using weighted residual procedures. The Galerkin method is also implemented for discrete representation with a mesh of tiny elements and Ni shape functions that are allocated to each facet of the mesh. An approximate Galerkin is achieved by combining heat transfer equation for the $\mathrm{XY}$ simulation model with $W=W(x)$ test function and integrating over the solution space $\Omega$. Same formulation is used for XZ simulation model by analogy.

$$
\int_{\Omega} W\left[\rho C_{p} \frac{\partial \tilde{T}}{\partial t}-\frac{\partial}{\partial x}\left(k \frac{\partial \tilde{T}}{\partial x}\right)-\frac{\partial}{\partial y}\left(k \frac{\partial \tilde{T}}{\partial y}\right)-q\right] d \Omega=0
$$

Then an approximate solution $\tilde{T}$ can be obtained,

$$
\tilde{T}(x)=\sum_{a=1}^{n_{\text {nodes }}} N_{a} \tilde{T}_{a}
$$

where number of element nodes is $n_{\text {nodes }}$.

The Galerkin method controls so that these functions obtained from the nodes are same as the solution:

$$
W(x)=\sum_{b=1}^{n_{\text {nodes }}} N_{b} W_{b}
$$

where the shape functions, $N_{b}$, are calculated at the node $\mathrm{b}$ for a given element as adjusted by constants $W_{b}$.

A matrix representation can be created as written below.

$$
\mathbf{C}(T) \dot{\mathbf{T}}+\mathbf{K}(T) \mathbf{T}=\mathbf{q}
$$

where heat capacity and heat conduction matrices are $\mathbf{C}(\mathrm{T})$ and $\mathbf{K}(\mathrm{T})$ respectively. The nodal temperature and rate vectors are $T$ and $\dot{\mathbf{T}}$ and the heat source is $q$. Furthermore, the heat capacity matrix is written as density and heat capacity dependent where $N$ is the shape function matrix.

$$
\mathbf{C}(T)=\int_{\Omega} \rho C_{p} \mathbf{N}^{T} \mathbf{N} \mathrm{d} \Omega
$$

Moreover, the heat conduction matrix is thermal conductivity dependent as given below.

$$
\mathbf{K}(T)=\int_{\Omega} k \mathbf{B}^{T} \mathbf{B} \mathrm{d} \Omega
$$

Finally, the heat source vector can be written as below.

$$
\mathbf{q}=\int_{\Omega} q \mathbf{N}^{T} \mathrm{~d} \Omega
$$

The element shape functions, $\mathbf{N}$, are second order iso-parametric triangles where, the variables $\xi$ and $\eta$ are two independent variables that describe the local coordinates of the nodes in each triangular element.

$$
\mathbf{N}=[\xi(2 \xi-1) 4 \xi 4 \xi(1 \xi-\eta) \eta(2 \eta-1) 4 \eta(1-\xi-\eta)(1-\xi-\eta)(2(1-\xi-\eta)-1)]
$$


The Gaussian quadrature time integration scheme is employed. The temperature dependent heat capacity and heat conduction matrices are recalculated at every simulation time step with an implicit approach as shown below.

$$
\mathbf{C}(T)\left(\frac{\Delta \mathbf{T}}{\Delta t}\right)+\mathbf{K}(T) \mathbf{T}^{\mathrm{t}+\Delta \mathrm{t}}=\mathbf{q}
$$

In order to capture the phase changing time steps between solidus and liquidus temperatures, the time increment, $\Delta t$, is fixed small enough for convergence. The equation systems is computed in an iterative loop at each time step using the NewtonRaphson method for capturing the temperature rise with the formulation below.

$$
\Delta \mathbf{T}=\left(\Delta t \times\left(\mathbf{C}^{-1} \mathbf{K}\right)+\mathbf{I}\right)^{-1}\left(\Delta t \times \mathbf{C}^{-1}\left(\mathbf{q}-\mathbf{K T}^{t}\right)\right)
$$

The next temperature temporal variable is computed after solving the temperature rise.

$$
\mathbf{T}^{\mathrm{t}+\Delta \mathrm{t}}=\mathbf{T}^{\mathrm{t}}+\Delta \mathbf{T}
$$

Then, the residual vector $\mathrm{R}$ is computed for comparison with an error tolerance:

$$
\mathbf{R}=\left(\Delta t\left(\mathbf{C}^{-1} \mathbf{K}\right)+\mathbf{I}\right) \times \Delta \mathbf{T}-\left(\Delta t \times \mathbf{C}^{-1}\left(\mathbf{q}-\mathbf{K} \mathbf{T}^{t}\right)\right)
$$

\subsection{Solution domain definition}

The physics-based XY and XZ simulations models with finite element meshes are shown together with underlying assumptions Figure 4 . The dimensions of the powder layer are 1 $\mathrm{mm}$ in length, $0.6 \mathrm{~mm}$ in width and $0.2 \mathrm{~mm}$ in thickness. The laser beam moves along a $0.5 \mathrm{~mm}$ long path. The mesh size in the finite element is 6292 with triangular elements for the XY simulation model and 2192 for the XZ simulation model with element size around $8 \mu \mathrm{m}$. The adiabatic boundary conditions are employed in the XY simulation model where these adiabatic boundaries are represented with $\Gamma 2, \Gamma 3, \Gamma 4$ and a fixed temperature boundary obtained from XY solution is represented with $\Gamma 1$.

The heat source vector $q(\mathrm{x}, \mathrm{y}, \mathrm{t})$ that is moving along the spatial variable $x$. The laser beam centre is moved by using the updated location of the spatial variable $x_{i+1}=v_{s} \Delta t+x_{i}$ where vs is the velocity of the laser beam scanning action during the L-PBF process. The heat source is distributed to the underlying elements in the mesh by keeping the energy density consistent. The Gaussian beam profile is used for the laser intensity variation.

A Dirichlet-type boundary condition is used for laser processing in the XZ simulation model where no heat source $(q=0)$ is applied. It is assumed that the spatial field on the $\mathrm{XZ}$ model is specified by setting $y=0$. The temperature on the location of $z=0$ in the XZ simulation model, a fixed boundary $\Gamma 1$ is set equal to the temperature attained from the $\mathrm{XY}$ simulation result.

$$
\left.T_{X Z}(x, z=0, t)\right|_{y=0}=\left.T_{X Y}(x, y=0, t)\right|_{z=0}
$$

This combining approach during the $\mathrm{XZ}$ simulation model is implemented and run after a temporal and spatial solution at the powder surface is achieved. Same adiabatic Г2, Г3 
and $\Gamma 4$ boundaries are employed in the $\mathrm{XZ}$ simulation model and an initial temperature of $T(x, z, t=0)=353 \mathrm{~K}$ is seeded throughout the elements.

Figure 4 Physics-based simulation of L-PBF process: (a) XY simulation model and

(b) XZ simulation model (see online version for colours)

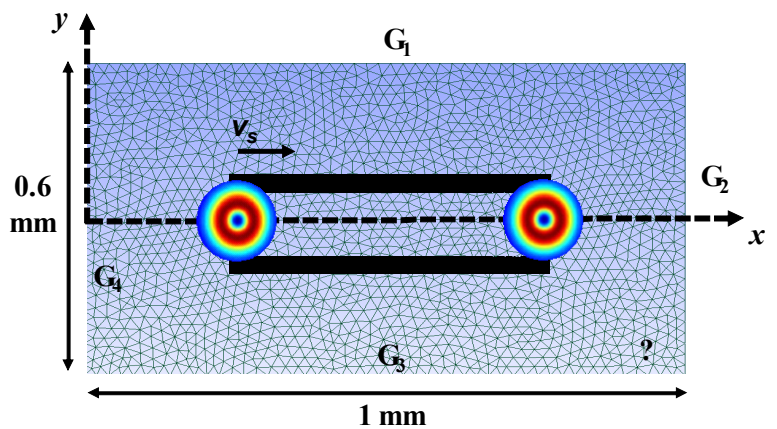

(a)

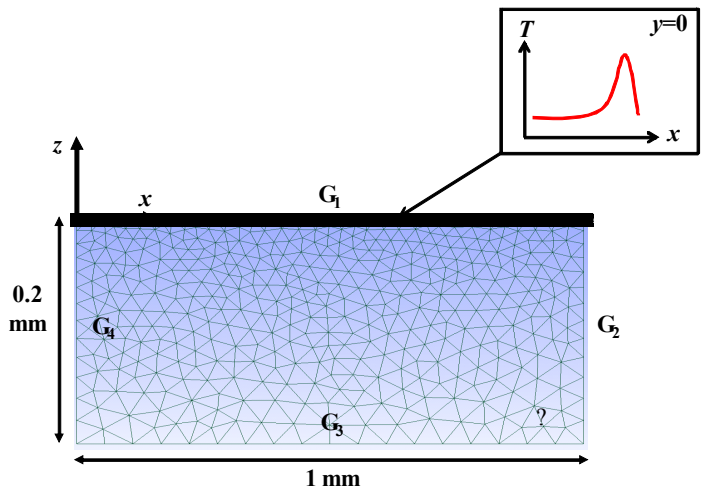

(b)

This solution domain definition together with the finite element simulation models are coded in MATLAB. The thermal fields of XY and XZ solution domains are utilised in deciding the melt pool geometry by using the melting point as the border for metal powder materials studied in this paper. The proposed simulation model is as practical as analytical models, while it does not consider complex physical interaction of liquid meltpool often possible to analyse with Computational Fluid Dynamics (CFD). However, it is much less time-consuming. For $\mathrm{XY}$ and $\mathrm{XZ}$ solutions and a laser scan length of 0.6 $\mathrm{mm}$, the simulation time on a PC computer with Intel Core i7 2600 processor and 16GB RAM is about $30 \mathrm{~min}$.

\section{Results and discussion}

The outcome of these physics-based simulation models to obtain thermal field solution for calculating the elements on the mesh based on their temperatures as solid, liquid and solid, and liquid phase and determine melt pool geometry. The melt pool changes its geometry as laser beam moves where the powder material in the vicinity of the melt pool 
is re-melted melted, re-solidified, or not yet fused powder. The powder material regions which are not sufficiently heated and melted are considered as incomplete fusion regions. The molten regions are identified as locations where the temperature is higher than the liquidus temperature of powder material as computed through the $\mathrm{XY}$ and $\mathrm{XZ}$ simulation models.

In this paper, physics-based simulation model validation is performed by comparing the results against the results in published literature by Roberts et al. (2009) for Ti6Al4V, Hussein et al. (2013) for SS316L stainless steel and Yadroitsev et al. (2007) for IN625 as summarised in Table 2.

Table 2 Simulation parameters are from Hussein et al. (2013) for SS316L, Roberts et al. (2009) for Ti6A14V and Yadroitsev et al. (2007), Criales et al. (2016) for IN625 and others are from Azo Materials (2020), Mat Web, Material Property Data (2020) for SS316L, Boivineau et al. (2006), Criales and Özel (2017) for Ti6A14V and Special Metals Corp. (2020) for IN625

\begin{tabular}{lccc}
\hline Parameter & SS316L & Ti6Al4V & IN625 \\
\hline Liquidus temperature, $\mathrm{Tl}[\mathrm{K}]$ & 1713 & 1933 & 1623 \\
Solidus temperature, Ts $[\mathrm{K}]$ & 1663 & 1873 & 1563 \\
Solid density, $\rho[\mathrm{kg} / \mathrm{m} 3]$ & 8027 & 4450 & 8440 \\
Latent heat of fusion, $\mathrm{Lf}[\mathrm{kJ} / \mathrm{kg}]$ & 277 & 275 & 227 \\
Specific heat, Cp $[\mathrm{J} / \mathrm{kg} \mathrm{K}]$ & 450 & $0.611 \mathrm{~T}+332.26$ & $0.2437 \mathrm{~T}+338.98$ \\
Thermal conductivity, $\mathrm{k}[\mathrm{W} / \mathrm{m} \mathrm{K}]$ & 14.6 & $0.015 \mathrm{~T}-1.334$ & $0.015 \mathrm{~T}+5.331$ \\
Reflectivity, $\mathrm{R}$ & 0.7 & 0.75 & 0.7 \\
Powder bed thickness, $\mathrm{s}[\mu \mathrm{m}]$ & 1000 & 30 & 50 \\
Laser power, $\mathrm{P}[\mathrm{W}]$ & 100 & 120 & 50 \\
Spot size diameter, $\mathrm{d}[\mu \mathrm{m}]$ & 150 & 100 & 70 \\
Scanning speed, $\mathrm{vs}[\mathrm{mm} / \mathrm{s}]$ & 200 & 220 & 130 \\
\hline
\end{tabular}

\subsection{Simulation model for Ti6Al4V}

The powder material properties for Ti6Al4V and laser fusion process parameters utilised in this simulation model are listed in Table 2, identical values as described by Roberts et al. (2009). For powder packing density, a porosity fraction of $\tau=0.4$ is estimated. The powder material on the build platform is warmed to $353 \mathrm{~K}$. In this argument, the temperature propagation offered by Roberts et al. (2009) is moderately corroborated. These initial thermal field measurements on the powder surface are conducted experimentally by employing an infrared camera in Fischer et al. (2002). The powder material reflectivity is assumed to be around $R=0.75$. The results show slight mismatch to those reported by Roberts et al. (2009) as shown in Figure 5(a). Roberts et al. reported a maximum temperature on the surface of approximately $2000 \mathrm{~K}$ whereas the peak temperature on the surface is found as $2080 \mathrm{~K}$ this physics-based simulation model. This is considered acceptable despite the fact that the heat loss due to conduction into the powder bed (z-direction) is not contemplated. Liu et al. (2021) reported a relative error as high as $37.3 \%$ in melt pool width predictions with their analytical model when the scanning speed (vs) is as low as $0.2 \mathrm{~m} / \mathrm{s}$ and the laser power $(P)$ is as high as $250 \mathrm{~W}$. 
The melt pool width was measured as $88.4 \mu \mathrm{m}$ at $P=50 \mathrm{~W}$, vs $=0.4 \mathrm{~m} / \mathrm{s}$ and $161.8 \mu \mathrm{m}$ at $P=250 \mathrm{~W}, \mathrm{vs}=1 \mathrm{~m} / \mathrm{s}$. The lowest mismatch of the melt pool width was determined to be as $6.8 \%$. The simulation model predicts with less than $10 \%$ melt pool width in this paper which is on par with predictions by Liu et al. (2021).

Figure 5 Temperature field and melt pool geometry for Ti6Al4V material: (a) as compared with Roberts et al. (2009) and (b) melt pool prediction. Blue: $\mathrm{T}<\mathrm{Ts}$; Yellow: Ts $<\mathrm{T}<\mathrm{Tl}$; Red: $\mathrm{T}>\mathrm{Tl}$ (see online version for colours)

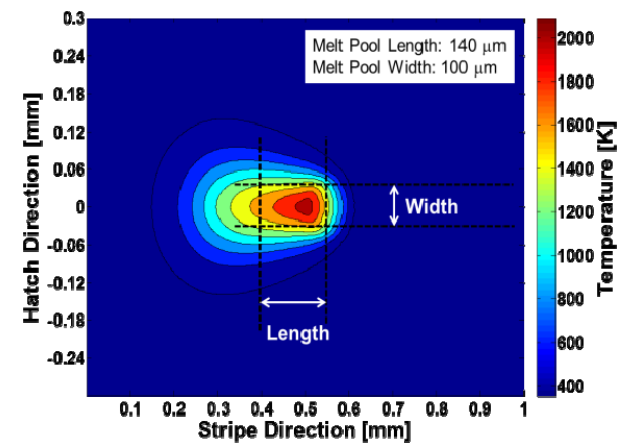

(a)

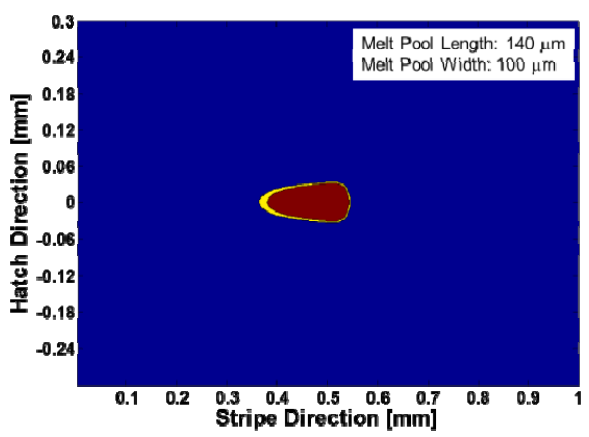

(b)

Furthermore, the simulation model offered is able to identify the melt pool dimensions at any given time by comparing the temperature distribution found previously with the solidus and liquidus temperatures of Ti6A14V, as shown in Figure 5(b). The powder material that is completely melted is indicated with in darker red brown colour. The powder material in the solidus + liquidus phase is presented with lighter yellow colour. Lastly, the unmelted powder material with temperature below the solidus temperature is indicated with blue coloured field. The benefit of producing results in this fashion is that the melt pool geometry is easily noticed. It should be noted that region below the solidus temperature may be previously melted and re-solidified due rapid heating and cooling rates due to intrinsic L-PBF process characteristics. By superimposing melt pool geometry and its time-dependent history, it is possible to obtain estimated track/hatch geometry and detect the sections re-melted and re-solidified.

The advantage of simulation-based digital twin is that the melt pool geometry can be tracked by studying the peak temperature reached by a node throughout the simulation result which creates a convenient link between powder properties and L-PBF process parameters and predicting the built quality by carefully observing the temperature field solution using some machine learning type intelligent approaches.

In addition, the temperature evolution for a stationary point in the thermal solution field is obtained as shown in Figure 6. For example, three stationary locations are sufficient to reveal how the temperature rises above the liquidus temperature (overmelting) and later declines from this peak (rapid cooling and solidification) using digital twin simulation results.

The overmelting time above the liquidus temperature, $t_{m}$, is critical for determining the onset of nucleation and hence solidification microstructure formation. The effect of latent heat of fusion is also noticeable in the cooling mechanism, as the time between the solidus temperature and liquidus temperature is slightly longer. 
Figure 6 Temperature evolution for L-PBF of Ti6Al4V at the powder surface $(z=0)$, along the laser centreline $(y=0)$, for three stationary locations $(x=0.35 \mathrm{~mm}, x=0.50 \mathrm{~mm}$, and $x=0.65 \mathrm{~mm})$ (see online version for colours)

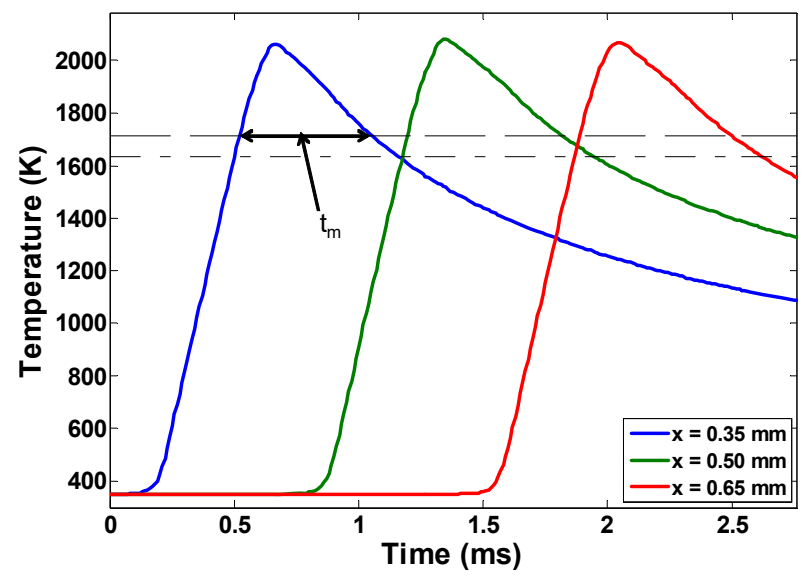

\subsection{Simulation model for SS316L}

The simulation model corroboration is performed for the L-PBF processing conditions on 316L stainless steel powder material as described by Hussein et al. (2013). The powder material properties and laser fusion process parameters used for this simulation model are reported in Table 2. For the SS316L material, a porosity fraction of $\tau=0.4$ is considered and the powder material is heated up to $353 \mathrm{~K}$ on the build plate.

The simulation results are shown in Figure 7 where a temperature field solution is obtained using the conditions suggested by Hussein et al. (2013). In their study, Hussein et al. (2013) presented an approximately $2600 \mathrm{~K}$ peak temperature on the powder surface whilst the physics-based simulation model of this study found a peak surface temperature of $2568 \mathrm{~K}$ as a close agreement between two findings for SS316L material. Hussein et al. (2013) further predicted melt pool dimensions using their temperature field solution.

Figure 7 Temperature filed (a) and melt pool geometry (b) obtained using the simulation model when compared against the melt pool prediction (inset) at powder surface by Hussein et al. (2013) (see online version for colours)

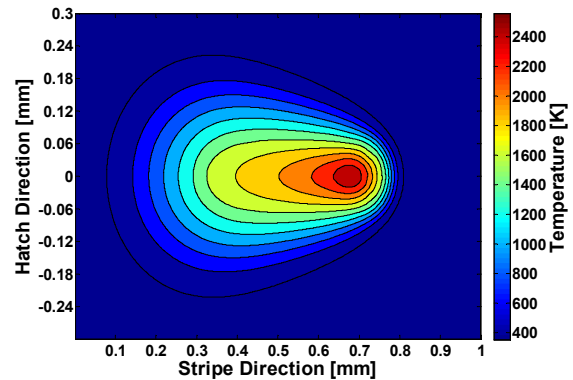

(a)

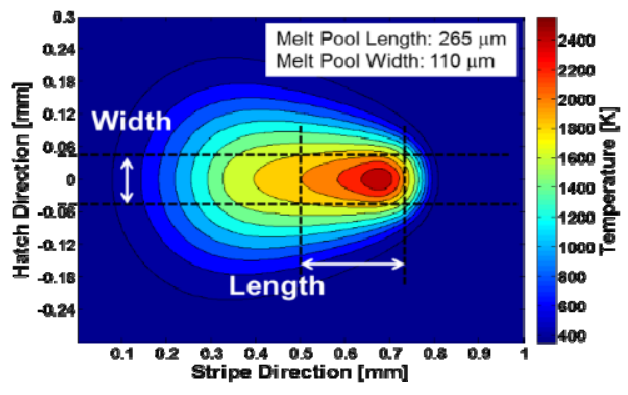

(b) 
The simulation model also gives for SS316L material the temperature profiles along the laser beam centreline for three stationary locations. At the beginning $(x=0.35 \mathrm{~mm})$, middle location $(x=0.50 \mathrm{~mm})$ and end of the processed track $(x=0.65 \mathrm{~mm})$ as shown in Figure 8 . The overmelting time above the melting temperature, $t_{m}$, is quite large in this case relative to the overall processing time. This situation is perhaps due to the exceptionally high temperatures attained while processing SS316L material under such laser fusion conditions. This observation confirms that the physics-based simulation model can be used for detecting regions of possible overheating and overmelting towards achieving process optimisation in a digital twin setting.

Figure 8 Temperature evolution for L-PBF of SS316L at the powder surface $(z=0)$, along the laser centreline $(y=0)$, for three stationary locations $(x=0.35 \mathrm{~mm}, x=0.50 \mathrm{~mm}$, and $x=0.65 \mathrm{~mm}$ ) for conditions used by Hussein et al. (2013) (see online version for colours)

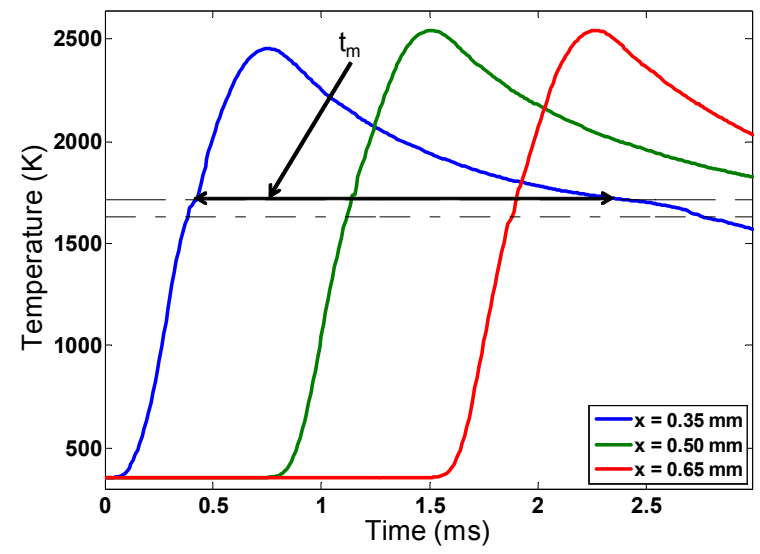

\subsection{Simulation mode for IN625}

The final simulation model is performed on IN626 nickel-based alloy powder material for the laser fusion process conditions reported by Yadroitsev et al. (2007). The properties of powder material and laser fusion process parameters used for this simulation model are given in Table 2. Similarly, a porosity fraction of $\tau=0.4$ is employed and the powder material on the build platform is preheated to $353 \mathrm{~K}$.

The thermal field solution in this case is given in Figure 9 that shows the temperature distribution obtained by the simulation model at the condistions used by Yadroitsev et al. (2007). The melt pool dimension for the XY and XZ simulations based on the liquidus temperature of IN 625 are also reported in the same simulation results.

The evolution of temperature rises and declines at three stationary points in the thermal solution field is obtained as shown in Figure 10. These stationary locations show that the peak temperatures reached to $2100 \mathrm{~K}$ and the time above the liquidus temperature is about $1.5 \mathrm{~ms}$ for laser fusion of IN625 material again providing a digital twin for improving process parameter optimisation in L-PBF based additive manufacturing. 
Figure 9 Physics-based simulation prediction for the melt pool geometry in L-PBF of IN 625:

(a) on the powder surface (XY field) and (b) longitudinal section (XZ field) (see online version for colours)

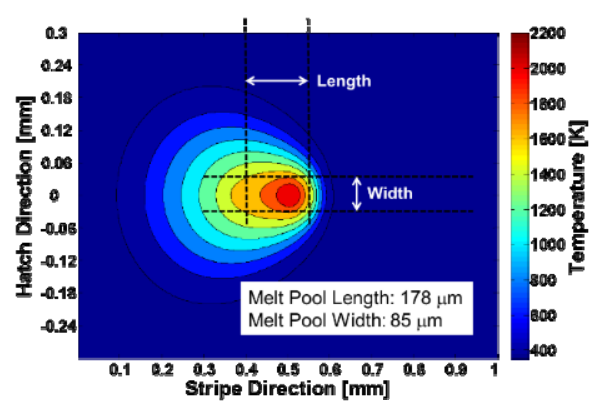

(a)

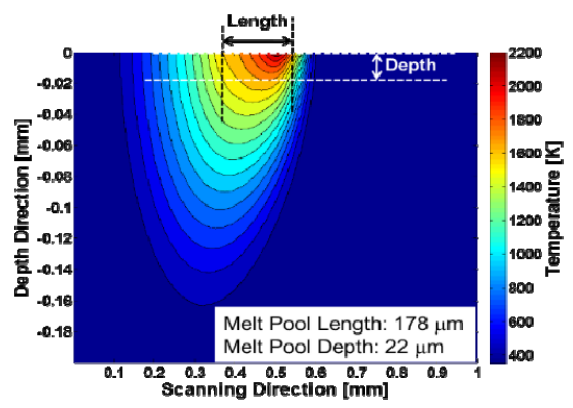

(b)

Figure 10 Temperature evolution for L-PBF of IN625 at the powder surface $(z=0)$, along the laser centreline $(y=0)$, for three stationary locations $(x=0.35 \mathrm{~mm}, x=0.50 \mathrm{~mm}$, and $x=0.65 \mathrm{~mm}$ ) as compared against the melt pool geometry in Yadroitsev et al. (2007) (see online version for colours)

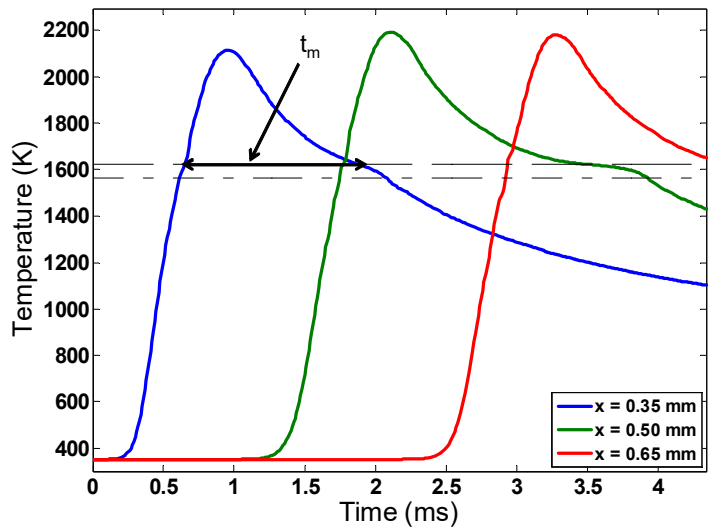

For comparison purposes, all simulation results are summarised in Table 3. The values given for peak temperature and overmelting time above melting temperature correspond to a specific location of the powder bed $(x=0.35 \mathrm{~mm}, y=0 \mathrm{~mm})$, or $100 \mu \mathrm{m}$ from laser centre starting point in the scanning direction along the track centreline. By singling out the locations in which the peak temperature is the highest, possible overheating zones can be identified in the physics-based simulation model and the digital twin. Similarly, locations in which peak temperature is below the desired level are useful indicators of the regions where incomplete fusion and associated defects may occur.

Table 3 Summary of simulation results at $x=0.35 \mathrm{~mm}(100 \mu \mathrm{m}$ from laser starting point $)$

\begin{tabular}{lcc}
\hline Material & Peak temp & Time above $T_{l}$ \\
\hline Ti6A14V & $2062.0 \mathrm{~K}$ & $0.67 \mathrm{~ms}$ \\
SS316L & $2453.9 \mathrm{~K}$ & $2.41 \mathrm{~ms}$ \\
IN625 & $2112.5 \mathrm{~K}$ & $1.21 \mathrm{~ms}$ \\
\hline
\end{tabular}




\section{Conclusions and future directions}

Digital twin of additive manufacturing requires effective but practical simulation models about the physics of the powder bed fusion processes. In this paper, physics-based simulation models are developed using 2D FEM are utilised for thermal and melt pool geometry prediction for obtaining a digital twin that can be used to study the L-PBF of metal powders. The results of the simulation models have been compared against the results published in the existing literature. The published research results for L-PBF of powder metals are used for processing powder Ti6A14V, SS316L and IN625. By comparing the results obtained through proposed physics-based simulation models with those from the literature, the simulation model parameters are refined, and comparable results are provided for L-PBF of Ti6Al4V and SS316L. The temperature predictions in the XY- and XZ-fields are also provided in L-PBF of IN625 alloy. Due to the two-dimensional assumption of the simulation model, predicted temperature values are found to be slightly higher as conduction heat loss in the z-direction is not considered for the initial XY simulation which is the base for the XZ simulation model. Additionally, the melt pool geometry characterisation is provided based on the temperature distributions obtained through the physics-based simulation approach. This prediction of the melt pool geometry can be further utilised for predicting layer geometry, and further on for optimisation of process parameters such as overlapping factor of fused section based on dimensional quality. Finally, a complete temperature evolution profile is reported demonstrating how a temperature rise with respect to time for a specific location on the surface takes place. The overheating time above the liquidus temperature is identified as a major simulation finding of the L-PBF process. It is expected that such physics-based functional simulation models will accelerate the progress toward digital twin of metal additive manufacturing processes.

Significant progress has been made in analytical and physics-based numerical simulation models for L-PBF processes. However, there remain some additional challenges which creates many future trends and research opportunities.

- For new materials and alloys specifically developed for L-PBF, there will a need for experimental data to validate numerical models and virtually eliminate or minimise defects such as pores, inclusions, and lack of fusion etc.

- There is also a need to reduce model uncertainty so that the predictions become more robust and consistent. The reliability of physics-based simulations models must be investigated and improved.

- More intense development for machine learning techniques is needed by using modern artificial intelligence tools to complement the existing physics-based, analytical, and numerical simulations so that they can be incorporated in the development of in-process and post-process quality improvement systems.

\section{References}

Abolhasani, D., Seyedkashi, S.M.H., Kang, N., Kim, Y.J., Woo, Y.Y. and Moon, Y.H. (2019) 'Analysis of melt-pool behaviors during selective laser melting of AISI 304 stainless-steel composites', Metals, Vol. 9, No. 8, p.876, https://doi.org/10.3390/met9080876 
Anam, M.A., Pal, D. and Stucker, B. (2013) 'Modeling and experimental validation of nickel-based super alloy (Inconel 625) made using selective laser melting', Proceedings of 23rd Annual International Solid Freeform Fabrication Symposium, University of Texas at Austin, pp.463-473.

Arisoy, Y.M., Criales, L.E. and Özel, T. (2019) 'Modeling and simulation of thermal field and solidification in laser powder bed fusion of nickel alloy IN625', Optics and Laser Technology, Vol. 109, pp.278-292.

Azo Materials (2020) Grade 316 Stainless Steel Datasheet, http://www.azom.com/properties.aspx? ArticleID=863 (Accessed June, 2020).

Bergheau, J.M. and Fortunier, R. (2008) Finite Element Simulation of Heat Transfer, J. Wiley \& Sons, Hoboken, New Jersey, USA.

Boivineau, M., Cagran, C., Doytier, D., Eyraud, V., Nadal, M.H., Wilthan, B. and Pottlacher, G. (2006) 'Thermophysical properties of solid and liquid ti-6Al-4V (TA6V) alloy', International Journal of Thermophysics, Vol. 27, No. 2, pp.507--529.

Criales, L., Arisoy, Y.M. and Özel, T. (2016) 'Sensitivity analysis of material and process parameters in finite element modeling of selective laser melting of Inconel 625', International Journal of Advanced Manufacturing Technology, Volume, Vol. 86, Nos. 9-12, pp.2653-2666.

Criales, L. and Özel, T. (2017) 'Temperature profile and melt depth in laser powder bed fusion of Ti-6Al-4V titanium alloy', Progress in Additive Manufacturing, Vol. 2, No. 3, pp.169-177.

Criales, L.E., Arisoy, Y.M., Lane, B., Moylan, S., Donmez, A. and Özel, T. (2017a) 'Predictive modeling, and optimization of multi-track processing for laser powder bed fusion of nickel alloy 625', Additive Manufacturing, Vol. 13, pp.14-36.

Criales, L.E., Arısoy, Y.M., Lane, B., Moylan, S., Donmez, A. and Özel, T. (2017b) 'Laser powder bed fusion of nickel alloy 625: experimental investigations of effects of process parameters on melt pool size and shape with spatter analysis', International Journal of Machine Tools and Manufacture, Vol. 121, pp.22-36.

Denlinger, E.R., Jagdale, V., Srinivasan, G.V., El-Wardany, T. and Michaleris, P. (2016) 'Thermal modeling of Inconel 718 processed with powder bed fusion and experimental validation using in situ measurements', Additive Manufacturing, Vol. 11, pp.7-15.

Fischer, P., Karapatis, N., Romano, V., Glardon, R. and Weber, H.P. (2002) 'A model for the interaction of near-infrared laser pulses with metal powders in selective laser sintering', Applied Physics A, Vol. 74, pp.467-474.

Gaikwad, A., Yavari, R., Montazeri, M., Cole, K., Bian, L. and Rao, P. (2020) 'Toward the digital twin of additive manufacturing: integrating thermal simulations, sensing, and analytics to detect process faults', IISE Transactions, Vol. 52, No. 11, pp.1204-1217, doi: 10.1080/ 24725854.2019.170175.

Gusarov, A.V. and Smurov, I. (2009) 'Two-dimensional numerical modelling of radiation transfer in powder beds at selective laser melting', Applied Surface Science, Vol. 255, pp.5595-5599.

Gusarov, A.V., Yadroitsev, I. and Bertrand Ph, Smurov I. (2007) 'Heat transfer modelling and stability analysis of selective laser melting', Applied Surface Science, Vol. 254, No. 4, pp.975-979.

Hussein, A., Hao, L., Yan, C. and Everson, R. (2013) 'Finite element simulation of the temperature and stress fields in single layers built without-support in selective laser melting', Materials and Design, Vol. 52, pp.638-647.

Jahn, M., Luttmann, A., Schmidt, A. and Paul, J. (2012) 'Finite element methods for problems with solid-liquid-solid phase transitions and free melt surface', Proc. Appl. Math. Mech., Vol. 12, pp.403-404.

Khairallah, S.A., Anderson, A.T., Rubenchik, A. and King, W.E. (2016) 'Laser powder-bed fusion additive manufacturing: physics of complex melt flow and formation mechanisms of pores, spatter, and denudation zones', Acta Materialia, Vol. 108, pp.36-45. 
Kolossov, S., Boillat, E., Glardon, R., Fischer, P. and Locher, M. (2004) '3-D FE simulation for temperature evolution in selective laser sintering process', International Journal of Machine Tools and Manufacture, Vol. 44, pp.117-123.

Liu, B., Fang, G. and Lei, L. (2021) 'An analytical model for rapid predicting molten pool geometry of selective laser melting (SLM)', Applied Mathematical Modelling, Vol. 92, pp.505-524, ISSN (0307)904X, https://doi.org/10.1016/j.apm.2020.11.027

Mat Web, Material Property Data (2020) 'AISI 316L stainless steel datasheet',(Accessed in June 2020) http://asm.matweb.com/search/SpecificMaterial.asp?bassnum=MQ316P

Mukherjee, T. and DebRoy, T. (2019) 'A digital twin for rapid qualification of 3D printed metallic components', Applied Materials Today, Vol. 14, pp.59-65(2019).

Ning, J., Sievers, D.E., Garmestani, H. and Liang, S.Y. (2019) 'Analytical modeling of in-process temperature in powder bed additive manufacturing considering laser power absorption, latent heat, scanning strategy, and powder packing', Materials., Vol. 12, No. 5, p.808, https://doi.org/10.3390/ma12050808

Ökten, K. and Biyikoglu, A. (2021) 'Development of thermal model for the determination of SLM process parameters', Optics and Laser Technology, Vol. 137, p.106825, https://doi.org/ 10.1016/j.optlastec.2020.106825

Özel, T., Altay, A., Donmez, A. and Leach, R. (2018) 'Surface topography investigations on nickel alloy 625 fabricated via laser powder bed fusion', International Journal of Advanced Manufacturing Technology, Vol. 94, Nos. 9-12, pp.4451-4458.

Patil, R.B. and Yadava, V. (2007) 'Finite element analysis of temperature distribution in single metallic powder layer during metal laser sintering', International Journal of Machine Tools and Manufacture, Vol. 47, pp.1069-1080.

Pauzon, C., Forêt, P., Hryha, E., Arunprasad, T. and Nyborg, L. (2020) 'Argon-helium mixtures as laser-powder bed fusion atmospheres: towards increased build rate of Ti-6Al-4V', Journal of Materials Processing Tech., Vol. 279, doi: 11655.10.1016/j. jmatprotec. 2019.116555.

Roberts, I.A., Wang, C.J., Esterlein, R., Stanford, M. and Mynors, D.J. (2009) 'A threedimensional finite element analysis of the temperature field during laser melting of metal powders in additive layer manufacturing', International Journal of Machine Tools and Manufacture, Vol. 49, pp.916-923.

Röttger, A., Boes, J., Theisen, W., Thiele, M., Esen, C., Edelmann, A. and Hellmann, R. (2020) 'Microstructure and mechanical properties of $316 \mathrm{~L}$ austenitic stainless steel processed by different SLM devices', Int. J. Adv. Manuf. Technol., Vol. 108, pp.769-783, https://doi.org/ 10.1007/s00170-020-05371-1

Schoinochoritis, B., Chantzis, D. and Salonitis, K. (2017) 'Simulation of metallic powder bed additive manufacturing processes with the finite element method: a critical review', Proc. Inst. Mech. Eng. Part, B.J. Eng. Manuf., Vol. 231, pp.96-117.

Song, B., Dong, S. and Liao, H. (2012) 'Process parameter selection for selective laser melting of ti6Al4V based on temperature distribution simulation and experimental sintering', Int. J. Adv. Manuf. Technol., Vol. 6, pp.967-974.

Special Metals Corporation (2020) Inconel 625 Datasheet, http://www.specialmetals.com/ documents/Inconel\%20alloy\%20625.pdf (Accessed in June 2020).

Van Elsen, M., Baelmans, M., Mercelis, P. and Kruth, J.P. (2007) 'Solutions for modeling moving heat sources in a semi-infinite medium and applications to laser material processing', International Journal of Heat and Mass Transfer, Vol. 50, pp.4872-4882.

Vasquez, F., Ramos-Grez, J.A. and Walczak, M. (2012) 'Multiphysics simulation of laser-material interaction during laser powder deposition', Int. J. Adv. Manuf. Technol., Vol. 59, pp.1037-1045. 
Yadroitsev, I., Krakhmalev, P. and Yadroitsava, I. (2014) 'Selective laser melting of ti6A14V alloy for biomedical applications: temperature monitoring and microstructural evolution', Journal of Alloys and Compounds, Vol. 583, pp.404-409.

Yadroitsev, I., Thivillon, L., Betrand, Ph. and Smurov, I. (2007) 'Strategy of manufacturing components with designed internal structure by selective laser melting of metallic powder', Applied Surface Science, Vol. 254, pp.980-983.

Yin, J., Zhu, H., Ke, L., Lei, W., Dai, C. and Zuo, D. (2012) 'Simulation of temperature distribution in single metallic powder layer for laser micro-sintering', Computational Materials Science, Vol. 53, pp.333-339. 